\title{
The Significance of Serum Tumor Markers CEA, CA19-9, CA125, CA15-3 And AFP in Patients Scheduled for Orthotopic Liver Transplantation: Do Elevated Levels Really Mean Malignancy?
}

Deniz Yavuz Baskiran ( $\sim$ dr.adde@hotmail.com )

inonu university turgut ozal medical center departmant of public helath

\section{Talha Sarigoz}

Kayseri City Hospital: Kayseri Sehir Egitim ve Arastirma Hastanesi

\section{Adil Baskiran}

liver trasnplantation

\section{Sezai Yilmaz}

liver transplantation

\section{Research Article}

Keywords: Chronic Liver Disease, Liver Transplantation, Hepacivirus, Hepatitis B virus, Biomarkers

Posted Date: December 30th, 2021

DOI: https://doi.org/10.21203/rs.3.rs-1193676/v1

License: (c) (1) This work is licensed under a Creative Commons Attribution 4.0 International License.

Read Full License 


\section{Abstract}

AIM AND BACKGROUND. Preparation of the patients for liver transplantation is a meticulous process and includes evaluation of tumor markers to rule out occult malignancy. Present study evaluated the significance of serum tumor markers in patients bound for liver transplantation due to viral and other etiologies of liver failure.

PATIENTS AND METHODS. 381 patientswho underwent liver transplantation were included in the study. Demographic data, Model for End stage.Liver Disease (MELD) scores and serum tumor marker levels were prospectively collected.

RESULTS: AFP levels were significantly higher in viral etiologies when compared to other etiologies $(p<0.05)$.Ca 19-9 was significantly higher in viral etiologies $(p<0.05)$. Among the viral etiologies HCV related liver failure had higher carcinoembryonic antigen (CEA) and Carbohydrate antigen 19-9 (Ca 19-9) levels $(p<0.05)$. A correlation was found between increasing MELD scores and serum levels of tumor markers $(p<0.05)$

CONCLUSIONS: Tumor markers such as AFP, CEA, Ca 125 and Ca 19-9 can be elevated in end stage liver disease. Their levels vary according to etiology and severity of disease. The diagnostic capabilities of these markers are reduced in end stage liver disease setting but they contribute to the evaluation of the pathophysiology of chronic liver disease. Transplantation can be performed safely in cases with high tumor marker levels provided that any occult malignancy is ruled out by means of imaging and endoscopic techniques. Tumor markers can guide the physician in determining the severity of liver cirrhosis and further studies are needed to validate such a relationship.

\section{Introduction}

Orthotopic liver transplantation (OLT) is the standard treatment for patients with end stage liver disease (ESLD). Chronic liver failure and liver cirrhosis is a risk factor for the development of certain malignancies such as hepatocellular carcinoma (HCC). In case of OLT to prevent any devastating results of a missed diagnosis of a malignancies certain precautions have to be taken. Therefore, evaluation of the patients with ESLD for OLT should be a meticulous process. Serum tumor markers including alpha feto protein (AFP), carcinoembryonic antigen (CEA), Carbohydrate antigen 19-9 (Ca 19-9), Carbohydrate antigen 15-3 (Ca15-3), Carbohydrate antigen 125 (Ca 125) are assessed to rule out any malignant process that may be concomitantly present ${ }^{1}$.

Viral hepatitis is the leading cause of ESLD that leads to OLT. Viral etiology shows differences according to the geographic distribution ${ }^{2-4}$.In our country HBV and HDV are the majiority of causes of ESLD. Another major etiology of ESLD is cryptogenic liver failure in our region ${ }^{5-7}$. It is a known fact that more than $50 \%$ of the HCC develop in the setting of HBV (either with or without HDV)related liver failure ${ }^{7}$ 
The main concern in the setting of acute or chronic liver failure is the reliability of the tumor markers for malignancy. Pissaia et al ${ }^{8}$ have reported increased levels of CEA, Ca 125, Ca15-3 and Ca 19-9 in patients being evaluated for liver transplantation and have founda correlation with the severity of the disease, presence of ascites and the etiology of ESLD (suchalcoholic cirrhosis etc.). In our region we specifically observe cryptogenic, HBV ad HDV related liver failure very frequently. Currently, there are no studies indicating the changes in serum tumor markers in patients with cryptogenic and viral related liver failure. Furthermore, the pattern of change of tumor markers in viral and cryptogenic liver failure bound for OLT in Turkish population is unclear

Our center is performing 300 cases of orthotopic liver transplantation per year due to various etiologies resulting in ESLD. We have a large data base that is prospectively formed regarding ESLD with various etiologies.The aim of our study was to evaluate the correlation of serum levels of AFP, Ca19-9, Ca125, Ca15-3 in patients with ESLD with various etiologies.

\section{Patients And Methods}

\section{Patient selection}

Between 2011 and 2016 a total of 1363 patients have undergone orthotopic liver transplantation in our clinic. The patients who were transplanted for hepatocellular carcinoma, who received a retransplantation and patients who lacked the study parameters in the preoperative work up and pediatric caseswere excluded from the study. A total of 381 patients were evaluated in the study.

All the patients received standard preoperative work up. Serum markers that consisted of CEA, Ca19-9, Ca 15-3, Ca125, AFP were preoperatively evaluated. In all cases malignant process was ruled out by imaging and endoscopic techniques.Demographic data and themodel for end stage liver disease (MELD) scores were collected. The diagnosis and the demographic data of the patients are given in Table 1. 
Table 1

Summary of the demographic data of the included in the study

\begin{tabular}{|c|c|c|c|c|c|}
\hline & $\begin{array}{l}\text { HBV } \\
(n=152)\end{array}$ & $\begin{array}{l}\mathrm{HCV} \\
(n=44)\end{array}$ & $\begin{array}{l}\mathrm{HBV}+\mathrm{HDV} \\
(\mathrm{n}=34)\end{array}$ & $\begin{array}{l}\text { Cryptogenic } \\
(n=151)\end{array}$ & $P$ value \\
\hline Female/male & $35 / 116$ & $25 / 19$ & $13 / 21$ & $58 / 93$ & $0.0001^{a}$ \\
\hline MeanAge (Years) & 49.5 & 57.5 & 47.7 & 52.7 & $0.0001^{b}$ \\
\hline MeanBMI $\left(\mathrm{kg} / \mathrm{m}^{2}\right)$ & 25.9 & 29.3 & 25.7 & 26.1 & $0.001^{c}$ \\
\hline MeanMELD score & 16 & 15 & 15 & 16 & 0.216 \\
\hline AFP elevation (\% of patients) & $27 \%$ & $31.8 \%$ & $41.2 \%$ & $5.3 \%$ & $0.0001^{d}$ \\
\hline CEA elevation (\% of patients) & $26.3 \%$ & $54.5 \%$ & $14.7 \%$ & $27.2 \%$ & $0.0001^{e}$ \\
\hline Ca125 elevation (\% of patients) & $58.6 \%$ & $75 \%$ & $58.8 \%$ & $65.4 \%$ & 0.06 \\
\hline Ca 19-9 elevation (\% of patients) & $53.9 \%$ & $68.2 \%$ & $52.9 \%$ & $36.4 \%$ & $0.0001^{f}$ \\
\hline Ca $15-3$ elevation (\% of patients) & $41.4 \%$ & $52.3 \%$ & $38.2 \%$ & $34.4 \%$ & 0.2 \\
\hline \multicolumn{6}{|c|}{$\begin{array}{l}\text { BMI= Body massindex }\left(\mathrm{kg} / \mathrm{m}^{2}\right) \text { MELD=Model forEndStageLiverDiseaseHBV: Hepatitis BVirus, HCV: } \\
\text { HepatitisCVirus, HDV: HepatitisD Virus, AFP: Alfa FetoProtein, CEA: Carcino-EmbryonicAntigen, Ca } \\
\text { 125: CarbohydrateAntigen 125, Ca 19-9: CarbohydrateAntigen 19-9, Ca 15-3: CarbohydrateAntigen } 15 \\
3\end{array}$} \\
\hline \multicolumn{6}{|c|}{$\begin{array}{l}\text { The variables are expressed as median (range). Tumor marker elevations are given as the percentage } \\
\text { of the patientswith elevated serum levels (i.e. serum value greater than the upper limit of the normal } \\
\text { range) }\end{array}$} \\
\hline \multicolumn{6}{|c|}{ a) Female gender most frequent in the HCV group when compared to other groups } \\
\hline \multicolumn{6}{|c|}{ b) Patients are older in the HCV group when compared to other groups $\backslash$} \\
\hline \multicolumn{6}{|c|}{ c) BMI is highest in HCV group when compared to other groups } \\
\hline \multicolumn{6}{|c|}{ d) Cryptogenic group has the lowest AFP levels when compared to other groups } \\
\hline \multicolumn{6}{|c|}{ e) HCV group has the highest CEA levels when compared to other groups } \\
\hline
\end{tabular}

\section{Evaluation of the Viral Markers}

Virologic assay was performed to all patients. HBV related surface antigen, "e" antigen, core antigen and anti-delta virus antibodies, anti-HCV antibodies wereall evaluated using the enzyme linked immunosorbent assay (ELISA; see below). Hepatitis B Virus Deoxyribonucleic Acid (HBV-DNA) and genotypes was determined by polymerase chain reaction (PCR)(COBAS AmpliPrep; Roche Diagnostics) and (INNOLIPA Genotyping assay; Fujirebio). Anti- HCV and anti-HBantibodies (all of them)were 
determined by ELISA (Vitros; Ortho Clinical Diagnostics). Hepatitis C Virus Ribonucleic Acid (HCV-RNA) levels were detected by PCR (COBAS AmpliPrep/COBAS TaqMan; Roche Diagnostics Systems).

\section{Evaluation of the tumor markers}

AFP, CEA, CA 15-3, CA 125, and CA 19-9 assays were performed using ELISA(Vitros; Ortho Clinical Diagnostics). The normal ranges for AFP, CEA, CA 15-3, CA 125, and CA 19-9 were 0-8, 0-5, 0-31.3, 0-35 and 0-35 UI/ $\mathrm{mL}$ (international units per milliliter)respectively.

\section{Statistical analysis}

The numeric data were expressed as median (range) deviationandpercentage. The continuous variables were compared using non-parametric tests(Mann-Whitney $\mathrm{U}$ test) among the grouping variables. Logistic regression was performed for correlation analysis and data are expressed as correlation coefficient (CC) and $p$ value with and $95 \%$ confidence interval $(\mathrm{Cl})$. Any $p$ value $\leq 0.05$ were considered significant. Statistical analyses were performed using Statistical Package for Social Sciences (SPSS version 22; IBM) Results

\section{Summary of the demographic data of the patients}

In total 381 patients were included in the study. The demographic data including age, gender and BMI were significantly different in the HCV group. Majority of the patients in the HCV group were female (56.8\%). Furthermore, patients in The HCV group had significantly older than the other groups [57.5(2379) years]. Median BMI in the HCV group was 25.7 (18-36) $\mathrm{kg} / \mathrm{m}^{2}$ which was higher than the other groups. MELD scores did not significantly change among the study groups. HBV, HBV+HDV and cryptogenic liver failure groups were comparable in terms of patient demographic. All the demographic data and MELD scores of the patients are summarized in Table 1.

\section{The changes in the level of the tumor markers according to the etiology of the liver failure}

AFP levels were elevated in only $5.3 \%$ of the patients with cryptogenic liver failure which was significantly lower than the other groups $(p=0.0001)$. CEA levels were elevated in $54.5 \%$ of the patients with HCV related liver failure which was higher than other study groups $(p=0.0001)$. Ca19-9 elevation was present in $68.2 \%$ of the patients with HCV related liver failure which was higher than the other study groups $(p=0.001)$. Other than these results there were no significant changes among the serum tumor markers in the other study groups. The percentage of the patients with elevated serum tumor levels among the study groups are summarized in Table 1.

\section{Elevation of the tumor markers according to the MELD scores of the patients}

The median MELD scores of the patients in the study was 17. The patients were grouped in to two groups according to their MELD scores. Patients with MELD scores $\leq 20$ constituted $76 \%$ of the patients $(n=290)$. The percentage of the patients with elevated serum tumor marker levels were evaluated among these two groups in order to study the effect of increasing MELD scores. The percentage of the patients with 
elevated Ca 125 were elevated with increasing MELD scores (62.4\% versus $74.7 \%, p=0.03)$. Percentage of patients with elevated Ca19-9 significantly increased with increasing MELD scores (45.2\% versus $59.3 \%$, $p=0.02$ ). Frequency of Ca 15-3 significantly increased with increasing MELD scores (36.6\% versus $45.9 \%$, $\mathrm{p}=0.03$ ). Other tumor markers did not change significantly with the MELD scores. All results regarding the elevation of the tumor markers according to MELD scores are summarized in Table 2.

Table 2

Evaluation of the serum tumor markers according to the MELD score of the patients.

\begin{tabular}{|llll|}
\hline & MELD $\mathbf{2 0}(\mathbf{n = 2 9 0 )}$ & MELD $>\mathbf{2 0}(\mathbf{n = 9 1 )}$ & P Value \\
\hline AFP elevation (\% of patients) & $20 \%$ & $20.9 \%$ & 0.86 \\
\hline CEA elevation (\% of patients) & $27.2 \%$ & $34.1 \%$ & 0.210 \\
\hline Ca125 elevation (\% of patients) & $62.4 \%$ & $74.7 \%$ & $\mathbf{0 . 0 3 1 ^ { \mathrm { a } }}$ \\
\hline Ca 19-9 elevation (\% of patients) & $45.2 \%$ & $59.3 \%$ & $\mathbf{0 . 0 2}$ \\
\hline Ca 15-3 elevation (\% of patients) & $36.6 \%$ & $45.9 \%$ & $\mathbf{0 . 0 3}^{\mathrm{b}}$ \\
\hline
\end{tabular}

The tumor markers are expressed of percentage of the patients with elevated serum level (i.e. serum value greater than the upper limit of the normal range).

MELD= Model for End Stage Liver DiseaseAFP: Alfa Feto Protein, CEA: Carcino-Embryonic Antigen, Ca 125: Carbohydrate Antigen 125, Ca 19-9: Carbohydrate Antigen 19-9, Ca 15-3: Carbohydrate Antigen 15-3.

a) Ca 125 levels we significantly higher in the patients with MELD score $>20$

b) Ca19-9 levels were significantly higher in the patients with MELD scores $>20$

c) Ca 15-3 levels were significantly higher in the patients with MELD scores>20

Factors determining the tumor marker elevation in the study groups

The factors determining the AFP elevation in HBV group was age (CC:8.3, Cl: 95\%, $\mathrm{p}=0.004)$ and MELD scores of the patients (CC:8.6, Cl: $95 \%, p=0.0003$ ).

CEA elevation in the HBV group showed a linear correlation with the age of the patient (CC:8.6, Cl: $95 \%$, $p=0.003)$.

Ca19-19 in the HBV group showed linear correlation with age (CC:7.2, Cl:95\%, $\mathrm{p}=0.007)$ and MELD scores (CC:5.4, Cl:95\%, $\mathrm{p}=0.02$ ) of the patients. In HCV group Ca19-9 levels showed a linear correlation with the $\mathrm{BMI}$ of the patients (CC:4.4, Cl:95\%, $\mathrm{p}=0.04)$.

Ca 125 levels showed a linear correlation with age (CC:8.2, Cl:95\%, $p=0.004)$ and MELD scores (CC:4.5, $\mathrm{Cl}: 95 \%, p=0.03$ ) of the patients in the HBV group.In cryptogenic liver failure group Ca 125 levels showed 
linear correlation with the age (CC:5.2, Cl:95\%, $\mathrm{p}=0.02)$ and MELD scores (CC:4.5, Cl:95\%, $\mathrm{p}=0.03)$ of the patients.

Ca 153 levels were significantly correlated with MELD scores (CC:4.3, Cl:95\%, $p=0.04)$ in HBV group and age (CC:3.9, $\mathrm{Cl}: 95 \%, \mathrm{P}=0$.) in the cryptogenic liver failure.

\section{Discussion}

It is very well known that cirrhosis, chronic HBV and HCV infection are risk factors for $\mathrm{HCC}^{9}$. We routinely use the tumor markers to check for any elevations that may guide us to focused surveillance for any occult malignancy during the evaluation of the patients with ESLD bound for OLT.However, in ESLD the diagnostic capability of tumor markers for biliary and gastrointestinal malignancies are debatable. Therefore, we perform a very detailed analysis in case abnormal serum tumor marker levels are detected.

Assmar et al ${ }^{10}$ found a correlation of serum tumor markers in patients with HBV and HCV that were newly diagnosed and were evaluated before starting an antiviral therapy. In their study, an elevation in Ca 125 levels in HBV infection. On the other hand, AFP had significantly increased in the serum of patients infected with HCV. However, the hepatic failure rate in the study was not reported and these patients were most probably in the beginning stages of their disease ${ }^{10}$. Usually correlation had been found between the tumor marker levels and disease severity. Evaluation of the levels of the tumor markers according to the etiology and stage of the liver disease is not clear. In our study, percentage of the patients with CEA, Ca19-9 were significantly higher in the HCV group and was indifferent among the other groups. Cryptogenic liver failure group showed the lowest frequency of AFP elevations.

Tumor markers and especially AFP has been studied in acute and chronic liver failure setting; specifically in the setting of hepatic malignancies ${ }^{10-13}$. AFP levels correlate with the regenerative capacity of the hepatoblasts and therefore were found to be increased after liver injury ${ }^{14}$. We observed lowest AFP levels in cryptogenic liver failure patients. These results suggest that reduced regenerative capacity of the liver in advanced stages of liver failure. We did not find any factor correlating with the levels of AFP in cryptogenic liver failure patients. However, AFP levels in HBV group were correlated with age and MELD scores of the patients which is generally the consistent with general literature ${ }^{12-14}$.

In our study it was found that CEA levels were highest in the HCV related liver disease. Our data is supported by the Maestranzi et al ${ }^{15}$ who have found highest CEA values in alcoholic and HCV related liver disease patients. Kitagawa et al ${ }^{16}$ co-localized CEA with proliferating cell nuclear antigen (PCNA) and found no overlap. This actually states that CEA is not related with the regenerative efforts of the hepatocytes but probably its expression is due to necrosis and inflammatory process directed by the inflammatory mediators. Since HCV causes enhanced inflammation and necrosis in both hepatocytes and bile duct cells, this may well be the underlying factor for CEA elevation in our study.Age was a significant factor affecting the CEA levels in HBV infected patients. 
Similarly, Singhal et al ${ }^{17}$ found that Ca 125 levels increased with increasing MELD score, ascites and alcoholic liver disease. We also observed increase in the frequency of CA 125 with increasing MELD scores of the patients. Ca 125 levels correlated with age and MELD scores of the patients with HBV related and cryptogenic liver failure. Defective protein clearance may be the reason for elevated levels of this tumor marker.

Bertino et al ${ }^{18}$ determined an increased Ca $19-9$ in HCV patients and Singhal et al ${ }^{17}$ determined an increase in Ca 19-9 levels with increasing severity of liver failure regardless of the etiology. We also found that the rate of elevated $\mathrm{Ca} 19-9$ was highest in HCV related liver failure. In our study we also found that with increasing MELD scores resulted an increase in the frequency of the patients with elevated Ca 19-9. This is most probably due to inflammatory destruction and fibrosis of the biliary system in the parenchyma observed specially in HCV related liver disease. Age, MELD scores and BMI correlated significantly with ca 19-9 levels in HBV and HCV groups in our study.

Ca 15-3 elevation was more prominent with increasing MELD scores in our study. The levels of ca 15-3 were correlated with age and MELD scores of the HBV related and cryptogenic liver failure.

Limitation of the present study includes the fact that it is a cross sectional study and the markers were not evaluated postoperatively over a time course. Furthermore we did not correlate or results with pathologic variables such as METAVIR score of the patients. Nevertheless; this study was performed in preoperative setting and liver biopsy is not routinely performed unless there is a clinical demand. The unique features of the present study that it is one of the largest studies that included patients with end stage liver failure bound for OLT in whom any malignant process had been ruled out by imaging and endoscopic techniques. Furthermore, our study showed that AFP levels with increasing MELD scores in patients with cryptogenic liver failure which is the first study to report such a pattern in cryptogenic and viral related liver failure.

In conclusion tumor markers such as AFP, CEA, Ca125 and Ca 19-9 can be elevated in end stage liver disease. Their levels vary according to etiology and severity of disease. The diagnostic capabilities of these markers are reduced in ESLD setting but they contribute to the evaluation of the pathophysiology of chronic liver disease. Transplantation can be performed safely in cases with high tumor marker levels provided that any occult malignancy is ruled out by means of imaging and endoscopic techniques. Tumor markers can guide the physician in determining the severity of liver cirrhosis and further studies are needed to validate such a relationship. As our patient cohort extend we will be able to give more detailed results and correlations regarding tumor marker elevations and the pathogenesis of liver failure.

\section{Declarations}

Disclosure statement: There is no conflict of ineteterest for the main and co-authors in the study

Funding: The authors declare that no funds, grants, or other support were received during the preparation of this manuscript. 
Competing Interests: The authors have no relevant financial or non-financial interests to disclose.

Ethics Approval: This retrospective study was conducted at Inonu University Institute of Liver Transplantation after obtaining institutional review board approval (2020/766).

\section{Author Information}

\section{Affiliations}

Departmant of Public Health, Inonu University, Turgut Ozal Medical Center , Malatya, Turkey Deniz Yavuz Baskiran

\section{Department of General Surgery, Kayseri City Hospital, Kayseri, Turkey}

Talha Sarigoz

Institute of Liver Transplantation, Inonu University, Turgut Ozal Medical Center , Malatya, Turkey

Adil Baskiran, Sezai Yilmaz

Author Contributions: All authors contributed to the study conception and design. Material preparation, data collection and analysis were performed by Deniz Yavuz Baskiran, Talha Sarigoz, and Adil Baskiran. The first draft of the manuscript was written by Deniz Yavuz Baskiran and all authors commented on previous versions of the manuscript. All authors read and approved the final manuscript.

\section{References}

1. Thuluvath PJ. Evaluation of Liver Transplant Recipients. J Clin Exp Hepatol. 2011;1:199-203.

2. Alqahtani S, Larson AM. Adult liver transplantation in the USA. Curr Opin Gastroenterol. 2011;27:240-7.

3. Burra P, et al. Liver transplantation for alcoholic liver disease in Europe: A study from the ELTR (European liver transplant registry). Am J Transplant. 2010;10:138-48.

4. Oketani $\mathrm{M}$, et al. Etiology and prognosis of fulminant hepatitis and late-onset hepatic failure in Japan: Summary of the annual nationwide survey between 2004 and 2009. Hepatol Res. 2013;43:97-105.

5. Kayaalp C, Ersan V, Yilmaz S. Acute liver failure in Turkey: a systematic review. Turkish J Gastroenterol. 2014;25:35-40.

6. Adil B, et al Hepatitis B Virus and Hepatitis D Virus Recurrence in Patients Undergoing Liver Transplantation for Hepatitis B Virus and Hepatitis B Virus Plus Hepatitis D Virus. Transplant. Proc.48, 2119-2123 (2016). 
7. ÖKTEN A. Türkiye'de Kronik Hepatit, Siroz ve Hepatosellüler Karsinoma Etiyolojisi. Güncel Gastroenteroloji. 2003;7:187-91.

8. Pissaia A, et al Significance of Serum Tumor Markers Carcinoembryonic Antigen, CA 19-9, CA 125, and CA 15-3 in Pre-Orthotopic Liver Transplantation Evaluation. Transplant. Proc.41, 682-684 (2009).

9. Tang A, Hallouch O, Chernyak V, Kamaya A, Sirlin CB. Epidemiology of hepatocellular carcinoma: target population for surveillance and diagnosis. Abdominal Radiology 1-13 (2017). doi:10.1007/s00261-017-1209-1.

10. Assmar M, Yeganeh S, Mansourghanaei F, Amirmozafari N. Combined Evaluation of AFP, CA15-3, CA125, CA19-9, and CEA Tumor Markers in Patients with Hepatitis B and C. Iran J Public Health. 2016;45:1645-51.

11. Jain SK, Rohatgi A, Raman KK, Sharma VK. Study of serum prealbumin and serum alpha fetoprotein in cases of fulminant hepatic failure. J Assoc Physicians India. 1995;43:462-3.

12. Takikawa Y, Suzuki K. Is AFP a new reliable marker of liver regeneration in acute hepatic failure? J Gastroenterol. 2002;37:681-2.

13. Varshney A, Gupta R, Verma SK, Ahmad S. Alpha-fetoprotein as a prognostic marker in acute liver failure: a pilot study. Trop Doct. 2016. doi:10.1177/0049475516653891.

14. Schiødt FV, et al. Alpha-fetoprotein and prognosis in acute liver failure. Liver Transpl. 2006;12:177681.

15. Maestranzi S, Przemioslo R, Mitchell H, Sherwood RA. The effect of benign and malignant liver disease on the tumour markers CA19-9 and CEA. Ann Clin Biochem. 1998;35:99-103.

16. Kitagawa $Y$, et al Immunohistochemical localization of CEA, CA19-9 and DU-PAN-2 in hepatitis C virus-infected liver tissues. Histopathology40, 472-479 (2002).

17. Singhal A, et al. Elevation of CA 125 and CA 19-9 in patients with end-stage liver disease. Int J Biol Markers. 2012;27:e147-51.

18. Bertino G, et al. Carbohydrate 19.9 antigen serum levels in liver disease. Biomed Res. Int.2013, (2013). 